\title{
La cultura material en la educación física escolar: discurso y praxis
}

\author{
Material culture in school physical education: discourse and praxis
}

A cultura material na educação física escolar: discurso e práxis

Flávia Carneiro Franco ${ }^{\mathrm{I}}$

\begin{abstract}
Resumen
Pretendemos mostrar el vínculo entre el Proyecto Educativo de Centro y la cultura material de la EF. Adoptamos el método etnográfico mediante un estudio de casos en un colegio público y en otro concertado de Burgos, España. Observamos las clases de EF de un grupo de cada escuela durante un curso. Analizamos la lógica interna y externa de 510 situaciones motrices: 242 realizadas en el centro público y 268 en el concertado. Constatamos que prevalecen las prácticas con objeto, si bien aquellas sin objeto son significativas en el colegio concertado. Los materiales empleados son comprados, industriales y específicos. Sin embargo, en el centro público son coloridos y atractivos, mientras que en el concertado son multiusos, poco sofisticados y antiguos. En conclusión, la escuela burgalesa tiende a reproducir la sociedad de consumo. No obstante, el centro concertado se distingue del público en una cultura material menos presente y específica.
\end{abstract}

Palabras clave: Educación física escolar; Proyecto educativo de centro; Praxiología motriz; Cultura material

\begin{abstract}
We aim to show the relation between the educational project and the use of space in the motor situations practiced in physical education. We adopted the ethnographic method with case studies from a public and private school in Burgos, Spain. We observed the PE classes of a group in each of the schools during a year. We analyzed the internal and external logic of the 510 motor situations: 242 practiced in the public and 268 in the private center. We found that practices with object prevail, although those without object are significant in the private school. The materials are purchased, industrial and specific. However, in the public center they are colorful and attractive, while in the private they are multipurpose, unsophisticated and old. In conclusion, the Burgos school tends to reproduce the consumer society. However, the private school is distinguished from the public in a less present and specific material culture.
\end{abstract}

Keywords: School physical education; Educational project; Motor praxeology; Material culture

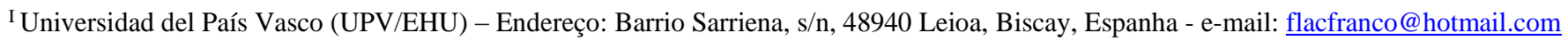




\section{Resumo}

Pretendemos mostrar a relação entre o projeto educativo e a cultura material da EF. Utilizamos o método etnográfico a partir de um estudo de casos em um colégio público e em outro privado de Burgos, Espanha. Observamos as aulas de EF de um grupo de cada escola durante um ano escolar. Analisamos as lógicas interna e externa de 510 situações motrizes: 242 realizadas no colégio público e 268 no privado. Constatamos que prevalecem as situações motrizes com objeto, embora aquelas sem objeto são significativas no colégio privado. Os materiais utilizados são comprados, industrializados e específicos. No entanto, no colégio público são coloridos e atrativos, enquanto no privado são polivalentes, pouco sofisticados e antigos. Concluímos que a escola de Burgos se inclina a reproduzir a sociedade de consumo. Contudo, o colégio privado se distingue do público em uma cultura material menos presente e específica.

Palavras-chave: Educação física escolar; Projeto educativo; Praxiologia motriz; Cultura material

\section{Introducción y marco teórico}

Las sociedades postindustriales se caracterizan por el uso masivo de materiales con diferentes fines para la realización de las actividades cotidianas (BAUDRILLARD, 1987; MORACE, 1993; WARNIER, 1999). Morace (1993) explica que la cultura material se ha transformado rápidamente durante el último siglo en las sociedades postindustriales. Se inventan diferentes tipos de objetos, cada vez más específicos, que además de satisfacer la función para la cual están destinados, han de ser estéticos y sofisticados para atraer al consumidor. Sin embargo, tienen una vida útil muy corta, por lo que son tirados a la basura y sustituidos regularmente por unos nuevos (MORACE, 1993). En el ámbito de la acción motriz, la supremacía de prácticas con objeto es evidente en la actualidad y una prueba de ello son los deportes olímpicos (PARLEBAS, 1999). Es el caso del fútbol, baloncesto, tenis, gimnasia rítmica, rugby, ciclismo y un centenar más de modalidades que se realizan con materiales altamente específicos.

Frente a las consecuencias negativas del consumismo, el discurso no consumista es frecuente en Ciencias de la Educación. Jiménez (1993) defiende la importancia de promover en el cotidiano escolar una actitud crítica de cara al consumismo, es decir, frente a "la actitud pasiva ante la sociedad de consumo centrada en el tener más y no el ser” (p. 9). Para la autora, más allá de discursos, una educación que pretenda formar ciudadanos críticos frente a las consecuencias negativas del consumismo habrá de aportar elementos que les permitan "situarse ante la sociedad de consumo como personas conscientes, críticas, responsables y solidarias" (p. 12), a través de "actuaciones educativas necesarias para crear actitudes de toma de conciencia, participación y actuación sobre la realidad concreta de la vida cotidiana de los alumnos y alumnas" (p. 12). Es necesario pensar en el conjunto de valores, normas y actitudes que derivan del currículo oculto y que “de una manera implícita conforman el microclima escolar” (p. 12).

La educación física también está cargada ideológicamente y sus actividades transmiten 
comportamientos y aptitudes sociales (PARLEBAS, 2001; KIRK, 2010; ETXEBESTE, 2012). De este modo, es necesario que las situaciones motrices estén vinculadas a las orientaciones pedagógicas, y se concreticen en programaciones y programas educativos que determinen la organización de las "situaciones de enseñanza sujetas a las exigencias del proyecto pedagógico de centro" (PARLEBAS, 2001, p. 148). En este sentido, la "profundización en la lógica interna de las distintas situaciones motrices permitirá apreciar con mayor claridad en qué medida cada una de ellas es capaz de satisfacer las finalidades establecidas" (PARLEBAS, 2001, p. 176).

Respeto al uso de materiales, el estudio de la lógica interna de las situaciones motrices nos permite diferenciarlas, en: situaciones motrices con objeto y situaciones motrices sin objeto (ETXEBESTE, 2012; ETXEBESTE, URDANGARIN, LAVEGA, LAGARDERA \& ALONSO, 2015; SANTEODORO, 2015; GIL, 2017; LÓPEZ DE SOSOAGA, 2017). Esta distinción nos permite examinar si prevalecen las situaciones motrices con o sin material, ya que frente a la hegemonía de las prácticas con objeto, la elección de situaciones motrices sin objeto podría ser acertada para ofrecer al alumnado un repertorio de este tipo de prácticas y favorecer una actitud no consumista en educación física.

Las situaciones motrices con objeto se caracterizan por el uso de uno o más materiales para su realización, si no, no existe contrato lúdico (ETXEBESTE, 2012; ETXEBESTE et al, 2015). Es inimaginable, por ejemplo, jugar al "campo quemado", a la "pelota sentada" o al fútbol sin un balón; andar en bicicleta sin una bicicleta o saltar a la comba sin una cuerda. En tales actividades el objeto marca la dinámica de la práctica y la transforma profundamente (PARLEBAS, 1999). Por ejemplo, para realizar un salto a la comba, el participante ha de mover las muñecas y antebrazos circularmente hacia adelante para que la comba de la vuelta, circule por encima de su cuerpo y la pueda saltar antes de que toque el suelo, evitando que se enrede en sus piernas, pies o tobillos y continúe rodando, siempre en la misma dirección.

En contraposición, existen situaciones motrices cuyas reglas no requieren el uso de objeto (ETXEBESTE, 2012). Es el caso de juegos como “zorros, gallinas y víboras”, "polis y cacos”, “pilla-pilla”, "la cadena", "muralla china" o "araña", de las carreras de resistencia o de velocidad, de los ejercicios de estiramientos o del taichí. En el "pilla-pila”, un jugador es el atrapador y los demás son jugadores libres. El atrapador trata de perseguir a los jugadores libres con el fin de pillar a uno con un toque y, así, intercambiar los roles; de tal forma que los jugadores no manipulan ningún objeto y sus acciones están marcadas por la conducta de los demás.

Del mismo modo, es fundamental reflexionar sobre la lógica externa de las situaciones motrices, ya que éstas se producen en "un contexto social determinado, y esa realidad social va a reflejarse en la actividad” (URDANGARIN, 2009, p. 5). La lógica externa está representada por los elementos sociales 
extrínsecos a las reglas de la situación motriz; es decir, sobre las costumbres no relacionadas con sus reglas (ETXEBESTE, 2012; ETXEBESTE, et al, 2015).

En primer lugar, identificamos si los materiales utilizados pertenecen a los centros o si el alumnado es responsable de aportar los objetos. En este último caso, la educación física incitaría a los estudiantes a tomar conciencia de la procedencia, de los medios de obtención o del precio de los materiales, y a situarse críticamente frente a la sociedad del consumo (JIMÉNEZ, 1993). Por una parte, los materiales pertenecientes a los centros son de fácil acceso para las clases de educación física. En el caso de que los maestros decidan emplearlos en las situaciones motrices propuestas, el alumnado no ha de aportar ningún material, todo está disponible. Por otra parte, los maestros pueden solicitar a los estudiantes que traigan materiales de casa: patines, patinetes, cuerdas o materiales desechados. Esta decisión por parte de la escuela incitaría al alumnado a responsabilizarse en contribuir con los materiales necesarios y, así, reflexionar sobre su origen, precio o medio de adquisición (BLÁNDEZ, 1995).

En cuanto al origen de los materiales utilizados en las situaciones motrices con objeto, éstos pueden provenir de un proceso de transformación industrial, pero también del medio natural, social o fabricados con materiales desechados (ETXEBESTE, 2012). Según Blández (1995) muchos materiales que se tiran a la basura, tales como periódicos, envases, tubos de cartón, sabanas o ropas viejas, pueden ser reutilizados para fabricar, por ejemplo, pelotas, picas, maracas, pañuelos o petos, y, así, el alumnado aprende a aprovechar materiales de desecho y a ir "tomando conciencia del significado de la sociedad de consumo" (p. 29). Así, con el fin de comprender el componente actitudinal relacionado con el cuidado del medio ambiente y con el consumo responsable, distinguiremos los materiales empleados en las situaciones motrices con objeto en: cogidos del entorno natural, obtenidos en el entorno social, fabricados por los jugadores y los que son fruto de un proceso de transformación industrial.

Los objetos cogidos del medio natural son aquellos que se presentan tal y como se encuentran en la naturaleza, por ejemplo, una piedra o una rama. Se trata de materiales que no provienen de un proceso de transformación y que no existen con la finalidad de jugar (ETXEBESTE, 2012). Los materiales obtenidos en el entorno social resultan de un proceso de transformación. Son normalmente comprados, aunque no estén fabricados para jugar sino que tienen otra función en la cultura. Es el caso, por ejemplo, de las chapas de botellas, que pueden ser empleadas en el "fútbol chapas" (ETXEBESTE, 2012). Los materiales fabricados por los jugadores son juguetes confeccionados con material desechado u obtenido en el entorno cultural o natural (ETXEBESTE, 2012). Por ejemplo, se pueden rellenar botellas de plástico y calcetines con arena para fabricar los bolos y la bola del juego de bolos. Los materiales provenientes de un proceso de fabricación industrial están manufacturados con un fin determinado (ETXEBESTE, 2012). En otros 
términos, están elaborados con una función específica, como pueden ser los altavoces, el plinto o un balón de vóley.

Finalmente, identificamos si los materiales utilizados en las situaciones motrices con objeto son específicos o no del área, ya que según su especificidad la escuela estará fomentando diferentes actitudes relacionadas con el consumo. En efecto, la escuela puede optar por reproducir la alta especificidad de los objetos en nuestra sociedad y en el ámbito físico-deportivo o, en cambio, servirse de materiales no específicos, sean éstos obtenidos de la naturaleza, del entorno cultural, fabricados por los jugadores o manufacturados con otro fin que el motriz. En este último caso, la institución educativa enseñaría al alumnado a aprovecharse de un abanico de materiales de diferentes características para las prácticas, en lugar de abocarle al uso de materiales específicos y comprados en tiendas especializadas. Por una parte, los materiales específicos son los balones de fútbol, baloncesto, balonmano o vóley, los aros, colchonetas y plintos, y una infinidad de objetos más. Según Etxebeste (2012) se tratan de juguetes o materiales deportivos que están fabricados para una actividad concreta, como el patinete, la peonza o el balón de fútbol. Son objetos comprados en tiendas especializadas y que provienen de un proceso de transformación industrial. De acuerdo con Blández (1995), son aquellos que "se vienen utilizando en las clases (...). Se compran en tiendas especializadas en material deportivo que suelen elaborar catálogos informativos con sus productos" (p. 24). Para la autora:

(...) muchos de estos elementos eran fabricados con materias primas naturales como la madera o la fibra vegetal y el colorido se reducía casi exclusivamente a su color natural y al verde. En la actualidad son fabricados, en su mayoría, con plásticos o gomas y con una gran variedad de colorido (BLÁNDEZ, 1995, p. 27).

Por otra parte, los materiales no específicos incluyen aquellos provenientes del entorno natural y cultural, los de fabricación propia o reciclados, y los manufacturados con un fin determinado, que no la práctica motriz, y que están comprados en otro tipo de tienda (BLÁNDEZ, 1995).

\section{Objetivos}

Este artículo trata de mostrar el vínculo existente entre la concepción desarrollada por el Proyecto Educativo de Centro de un colegio público y de otro concertado de la ciudad de Burgos (Comunidad de Castilla y León, España) y la cultura material de la educación física. Así, pretendemos:

- Poner de manifiesto la lógica interna y externa de las situaciones motrices realizadas en la educación física de cada escuela y captar los significados relacionados con la cultura material. 
- Mostrar la relación existente entre la cultura material de la educación física de cada escuela y los discursos pedagógicos presentados en las leyes educativas y en sus proyectos educativos.

\section{Metodología}

Este trabajo de investigación adopta el método etnográfico. La tradición etnográfica se caracteriza principalmente por estudiar en profundidad una cultura específica. En este sentido, los fenómenos sociales son entendidos "como particulares (...) más bien que como replicables y claramente definidos" (RUIZ OLABUENAGA \& ISPIZUA, 1989, p. 21). Peter Woods (1987) afirma que el uso de la etnografía en la escuela posibilita estudiar desde "dentro" y en profundidad diferentes aspectos que conforman la realidad escolar. Para el autor los estudios etnográficos en el ámbito educativo posibilitan revelar "capas de significación que permanecen ocultas a la observación superficial y que a menudo son diferentes de lo que se supone que son" (WOODS, 1987, p. 21).

En la presente investigación realizamos un estudio de casos en dos centros educativos representativos en la ciudad de Burgos, uno público y otro católico. Por un lado, un centro público con una larga trayectoria educativa, el Colegio Público Padre Manjón. Por otro lado, un centro concertado católico con más de un siglo de tradición, el Colegio Nuestra Señora de Lourdes. Esta elección nos permite comprender sus diferencias y similitudes, así como conocer el sistema educativo burgalés en sus dos ámbitos, público y privado, que comparten la educación de los jóvenes de esta ciudad. Asimismo, escogemos dos centros educativos que se sitúan en la misma zona de la ciudad con el fin de profundizar en un contexto específico.

En el estudio de los proyectos educativos buscamos el contenido manifiesto por los centros, en lo que hace referencia a sus discursos pedagógicos. Para ello, realizamos un análisis de contenido en el cual profundizamos en los términos y conceptos claves recurriendo a diccionarios y documentos de la orden franciscana para analizar el contenido declarado desde el punto de vista semántico y conceptual.

Por otra parte, recogemos la información relativa a la lógica interna y externa de las situaciones motrices mediante la observación participante. La observación es llevada a cabo a lo largo del curso lectivo 2009/2010: la iniciamos en septiembre del año 2009 y la finalizamos en junio de 2010. Acudimos a todas las clases de educación física de un sexto curso de primaria (equivalente al $7^{\circ}$ ano do Ensino Fundamental en Brasil) del centro público (65 clases de 50 o 55 minutos) y de un grupo del mismo nivel educativo del centro concertado (55 clases de 1 hora). Elaboramos un diario de campo compuesto por las observaciones 
realizadas. A la vez que observamos los hechos tomamos notas de las explicaciones del profesor, comentarios o dudas del alumnado, si bien la principal preocupación es recoger información correspondiente a las normas de las situaciones motrices realizadas. Luego, en el mismo día, describimos los acontecimientos observados y reconstruimos las normas de las situaciones motrices.

Para el análisis de la lógica interna y externa de las situaciones motrices observadas, se elabora una ficha de análisis que se centra en las características del espacio, las relaciones, el tiempo y los objetos de cada situación motriz. Recurrimos al diario de campo y describimos y analizamos 510 situaciones motrices: 242 practicadas en el centro público y 268 en el concertado.

Figura 1 - Ejemplo de ficha de análisis de la lógica interna y externa de las situaciones motrices estudiadas.

\section{Fecta: 061109}

CONSTRUCCIONES CON ZL CUERPO EX TRTOS - FIGURA 3

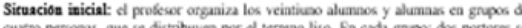
cuarto penceas, que se distribuyea per al ternea liso. En cadi grupoc dos portiors se

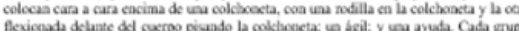

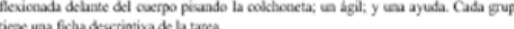

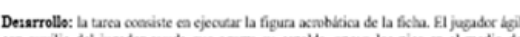

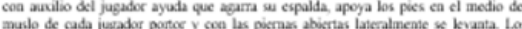

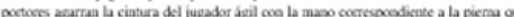

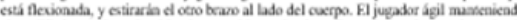

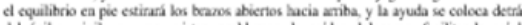

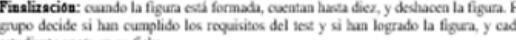

Antlisi Logica Externa

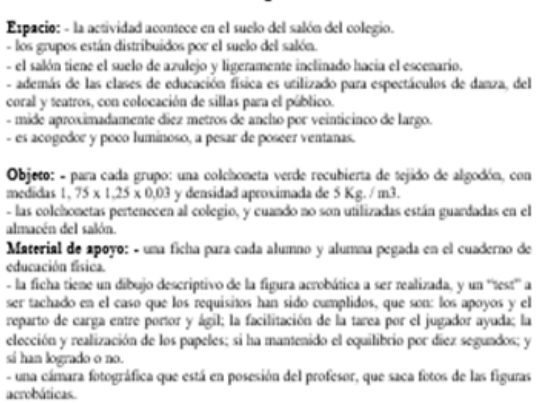

Participantes: - veintimo alumas y alumas de sexto cuno de pimaria

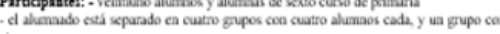

cinea.

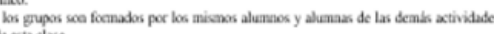

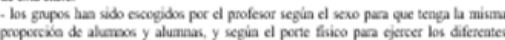
upciss ea la tares. Eero: micto

Dintatica sccial extramotrix: $: 0$

Tiempo: - La tares es pante de una de las actividado de una chase de edacacio flisia.

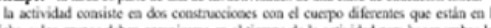

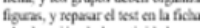

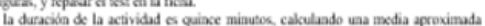

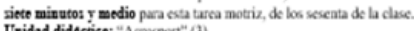

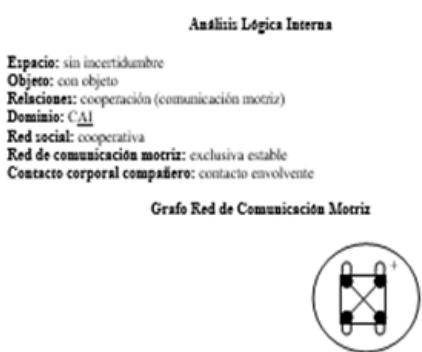

Red do isteraccion de marces uin marca

Red de roles: fijo

Memoris: sia memoria

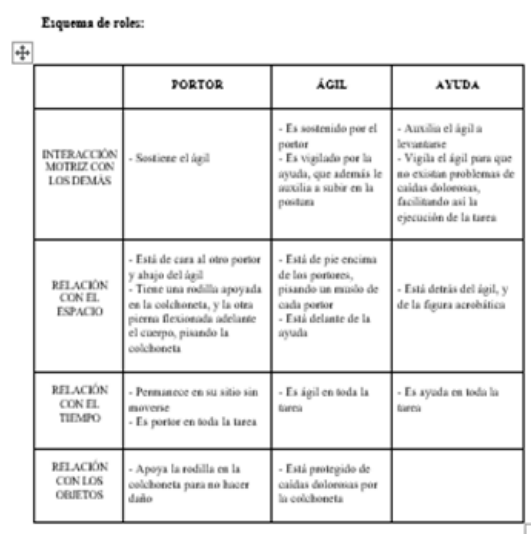


El tratamiento de la información obtenida mediante las fichas de análisis se realiza con el programa informático SPSS Statistics. Identificamos los porcentajes de las variables referentes a las categorías de la lógica interna y externa en función de la suma total del tiempo de práctica motriz. Así, la duración de cada una de las situaciones motrices observadas es la unidad de referencia. Para la interpretación de los datos seleccionamos las categorías de mayor interés, con el objetivo de revelar los rasgos de la lógica interna y externa que prevalecen en la educación física.

\section{Resultados}

El cambio climático, la devastación de los bosques, la depredación de los recursos naturales, la contaminación del aire y de los ríos y océanos son los efectos negativos del incremento del consumo y de la elevada industrialización. En consecuencia, la problemática ambiental se manifiesta como uno de los temas de mayor relevancia a nivel global y, más allá de los trabajos académicos, se ha convertido en la temática de cumbres internacionales, leyes y promesas políticas. A nivel internacional se imponen restricciones a los países desarrollados con el fin de reducir la producción y, así, las emisiones. La Unión Europea, a su vez, se encarga de asignar a los países miembros medidas a corto plazo, pero también a largo plazo de cara a mitigar el problema. De entre estas medidas, en el ámbito educativo destaca la implantación de competencias curriculares relacionadas con el cuidado del medio ambiente y con el consumo responsable.

\subsection{Discursos pedagógicos: consumo responsable y preservación de la naturaleza}

La Ley Orgánica 2/2006, de 3 de mayo, de Educación, decreto en vigor durante la etnografía, plantea la "Competencia en el conocimiento y la interacción con el mundo físico", que tiene como fin favorecer habilidades y destrezas relacionadas con "el uso responsable de los recursos naturales, el cuidado del medio ambiente, el consumo racional y responsable, y la protección de la salud individual y colectiva como elementos clave de la calidad de vida de las personas". Según la Ley Orgánica de Educación (LOE) esta competencia básica "supone asimismo demostrar espíritu crítico en la observación de la realidad y en el análisis de los mensajes informativos y publicitarios, así como unos hábitos de consumo responsable en la vida cotidiana".

Por su parte, las escuelas estudiadas presentan en sus proyectos educativos actitudes y valores relacionados con las competencias planteadas en la LOE. La escuela pública plantea la valoración, el respeto y el cuidado del patrimonio natural y sociocultural en sus valores relacionados con el entorno (CEIPPM, 2009), si bien no se dirige directamente a la cuestión del consumo responsable planteado por el 
Decreto ley. El centro católico, por su parte, refuerza en el valor "respeto" de su ideario la intención de favorecer el respeto, la valoración y el cuidado de la naturaleza, al tiempo que se posiciona firmemente frente a la problemática del consumo exacerbado, fundamentado en la sencillez y en la pobreza franciscana; muy evidentes, por ejemplo, en la norma "adoptar un estilo de vida no consumista: 'No es más feliz el que más tiene sino el que menos necesita" y en la actitud "capacidad para valorar el Ser sobre el Tener", planteada en el valor sencillez (CPCNSL, 2004). En cualquier caso, observamos como ambos centros reaccionan frente a la devastación de la naturaleza y plantean el uso responsable de sus recursos, si bien el centro público omite la cuestión del consumo responsable planteado por la LOE, el franciscano va más allá del Decreto y presenta claramente la intención de favorecer una actitud no consumista.

4.2 La cultura material en la educación física: objetos comprados, industriales y específicos

Los objetos utilizados en la educación física del Colegio Público Padre Manjón son propiedad del centro, comprados en tiendas especializadas en material deportivo y de fabricación industrial. Asimismo, son, en su mayoría, coloridos y atractivos.

Figura 2 - El alumnado utiliza un stick y una bola para jugar al hockey en la cancha polideportiva de la escuela pública durante una clase de educación física.

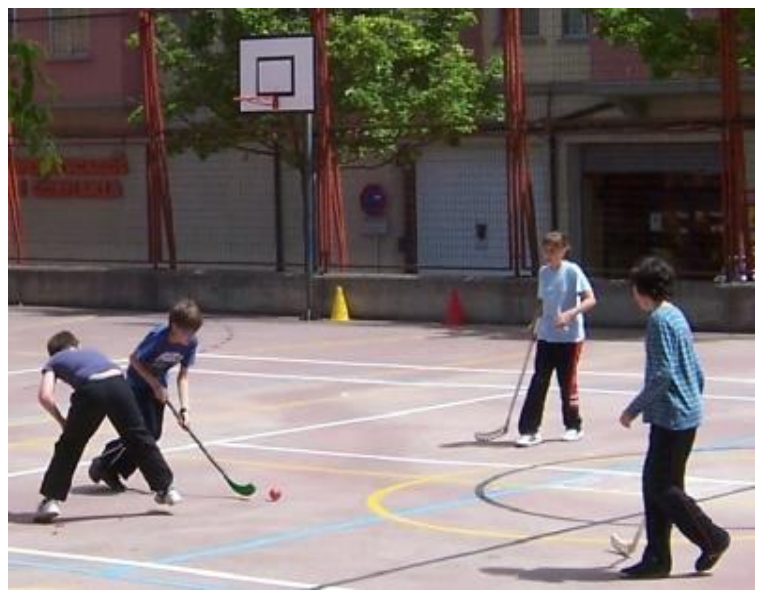

El estudio de la lógica interna muestra que las situaciones motrices con objeto representan el 73,4\% del tiempo de práctica (1640 minutos). El alumnado práctica carreras de relevo, actividades de saltar los bancos suecos, los juegos como "campo quemado", “Alien”, “a dar más vueltas", "mariposas”, "barco pirata", "alturitas" o "sanfermines", deportes colectivos, entre otras situaciones motrices con objeto. En “alturitas", por ejemplo, los jugadores libres pueden subirse a las espalderas o a uno de los bancos suecos esparcidos por el tatami del gimnasio para convertirse en "invulnerables". El atrapador, a su vez, lleva un balón de goma, parcialmente deshinchado, para tocar a los jugadores libres. En el caso del "campo quemado", los estudiantes tratan de golpear a un adversario con un balón de plástico para convertirle en 
atrapado, es decir, mandarle al cementerio. Si un jugador encarcelado golpea a un adversario, le convierte en prisionero y vuelve a su campo, está otra vez libre.

Figura 3 - Un estudiante se dirige al cementerio portando el balón de plástico, tras ser golpeado en el juego "campo quemado", durante una clase de educación física en el gimnasio del colegio público burgalés.

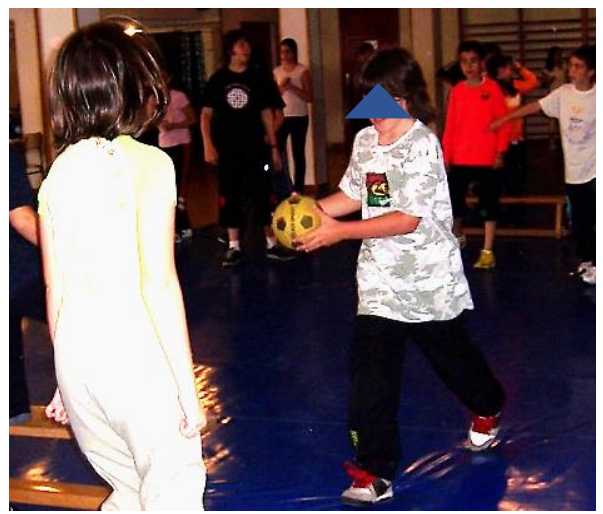

En cambio, las situaciones motrices sin objeto suponen el 26,6\% del tiempo (594 minutos). El alumnado del colegio público de Burgos participa, por ejemplo, en los juegos "muralla china", "gato y ratón" o "Tarzan y los leones", en los ejercicios de estiramientos, del yoga, en las carreras de resistencia y de velocidad, o realizan un tramo del Camino de Santiago sin objeto.

Figura 4 - El grupo de sexto de primaria del colegio público burgalés realiza una marcha sin material en un tramo del Camino de Santiago.

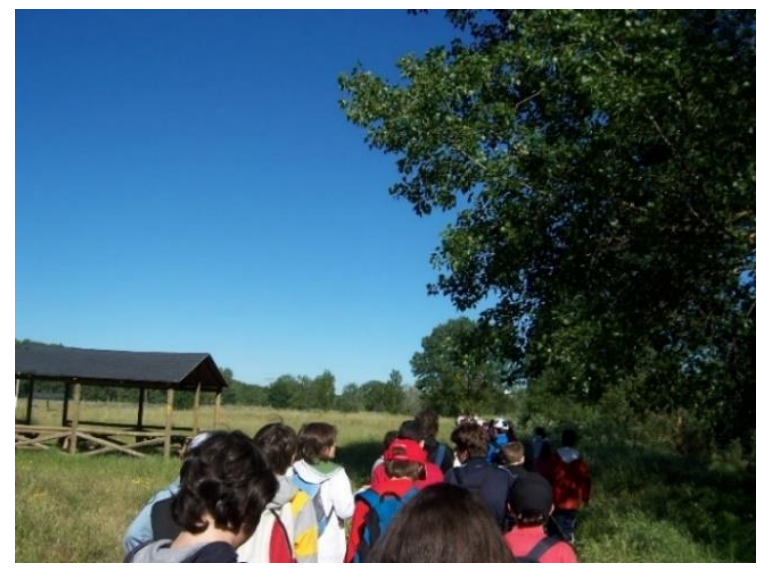

Por ejemplo, en "Tarzan y los leones", los "tarzanes" intentan atrapar a los "leones", mediante un simple toque, para convertirles igualmente en "tarzanes". Para desplazarse, tanto los tarzanes como los leones han de tocar el suelo con las manos. En el caso de "muralla china", los "atrapadores" permanecen en la línea central de la cancha, donde intentan atrapar a los jugadores libres para convertirles en atrapadores. En el "dragón”, el jugador cabeza guía la cadena por la extensión del gimnasio y su intención es que algún jugador se desprenda de la cadena para intercambiar los roles. 
Figura 5 - ¿Se juega con o sin objeto en la EF del centro público?

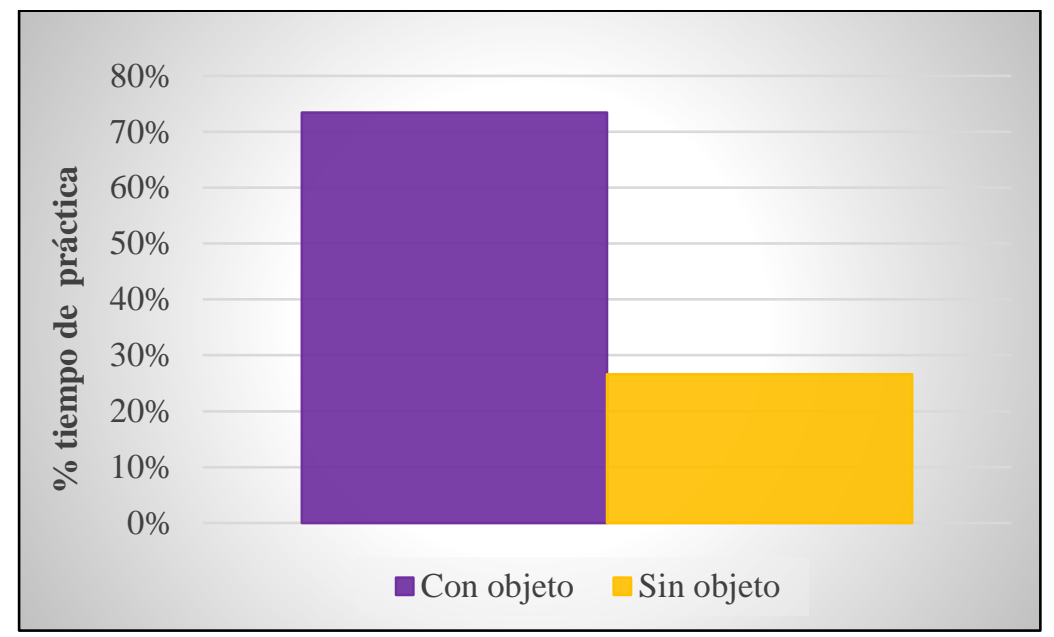

Las características de los objetos utilizados en el Colegio Nuestra Señora de Lourdes coinciden con aquellos empleados en el centro público. No obstante, son, en su mayoría, antiguos, poco sofisticados y multiusos.

Figura 6 - Un grupo de alumnos ejecuta una figura acrobática con la protección de una colchoneta en la superficie del salón de actos de la escuela franciscana burgalesa.

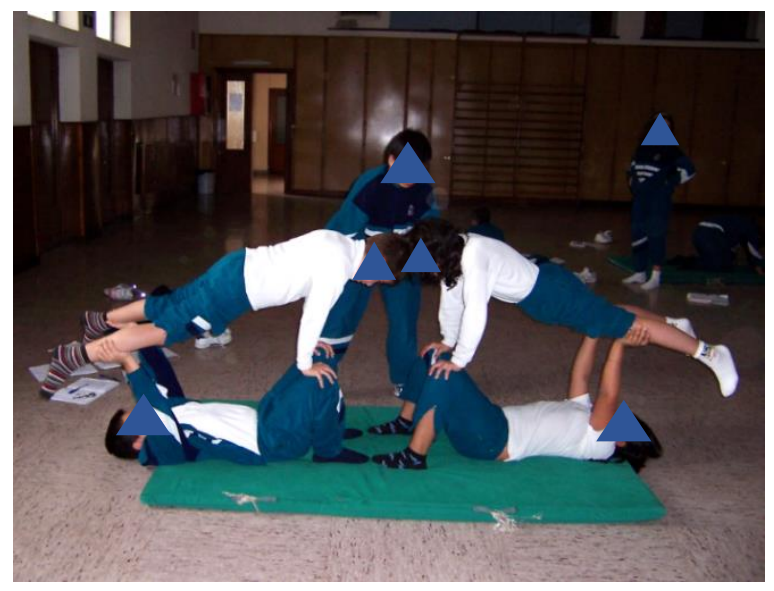

El estudio de la lógica interna revela que en el colegio concertado también predominan las situaciones motrices con objeto, pero su presencia es bastante inferior: representan el 65,7\% del tiempo de práctica (1536 minutos). Los estudiantes participan en el "balón torre”, “campo quemado", “juego con balón" o "bote bote", en las figuras acrobáticas, en las actividades "lazarillo" y en el "masaje con balón" o en la treintena de saltos a la comba. En el "bote bote", el atrapador vigila el bote evitando que los jugadores libres le den una patada. El "bote" es un balón de goma azul, parcialmente deshinchado, para no alejarse demasiado en el momento de la patada. En "balón torre", el equipo realiza pases con el balón de goma con el objetivo de hacerlo llegar a la torre, sin que el equipo adversario consiga recuperarlo. En el "juego con balón”, por su parte, el equipo realiza pases con el fin de meter el balón en la portería y marcar un punto. 
El equipo que defiende intenta interceptar el balón para recuperarlo a su equipo.

Figura 7 - Una alumna realiza una de las modalidades de salto a la comba individuales en el salón del centro franciscano burgalés.

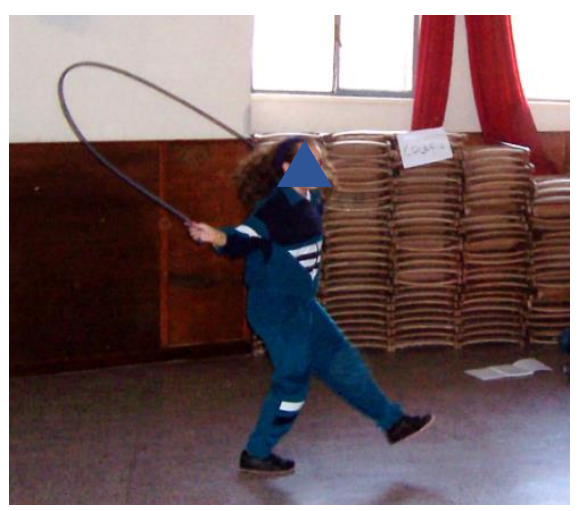

Por su parte, la práctica de situaciones motrices sin objeto es superior al centro público y representa el 34,3\% del tiempo de práctica (800 minutos). El alumnado participa de los ejercicios de calentamiento y estiramientos, de los contrabalanceos en parejas, de duelos individuales tales que "guerra de pulgares", “calientamanos", "pulso de pie", "sumo de rodillas", "pelea de gallos" o "Pepo y Pepito", de los juegos como "la araña", "polis y cacos" o "zorros, gallinas y víboras" o de las carreras de resistencia sin utilizar material alguno.

Figura 8 - Dos estudiantes juegan al "calientamanos" en la superficie del salón del centro franciscano burgalés.

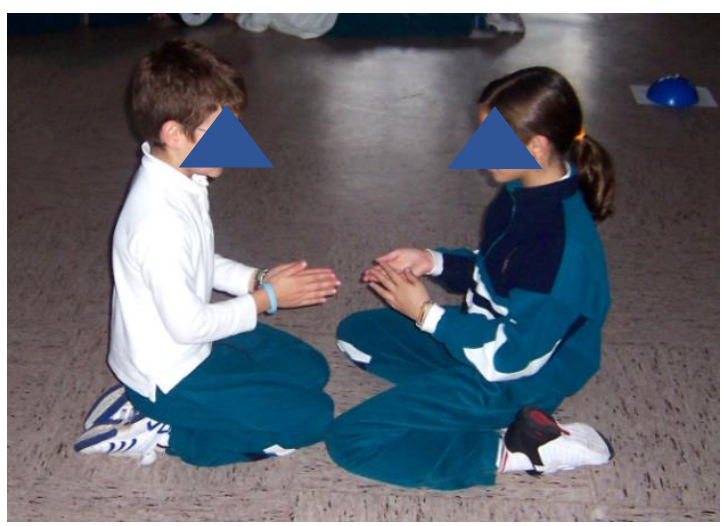

Por ejemplo, en los ejercicios de calentamiento y estiramientos que el maestro propone al principio y final de la clase, los estudiantes tienen que repetir de forma conjunta la secuencia de acciones a manos libres que éste determina. En el caso del juego "la araña", los atrapadores intentan tocar a los jugadores libres, que cruzan la tela imaginaria de un lado a otro, para convertirles en tela. 
Figura 9 - ¿Se juega con o sin objeto en la EF del centro concertado?

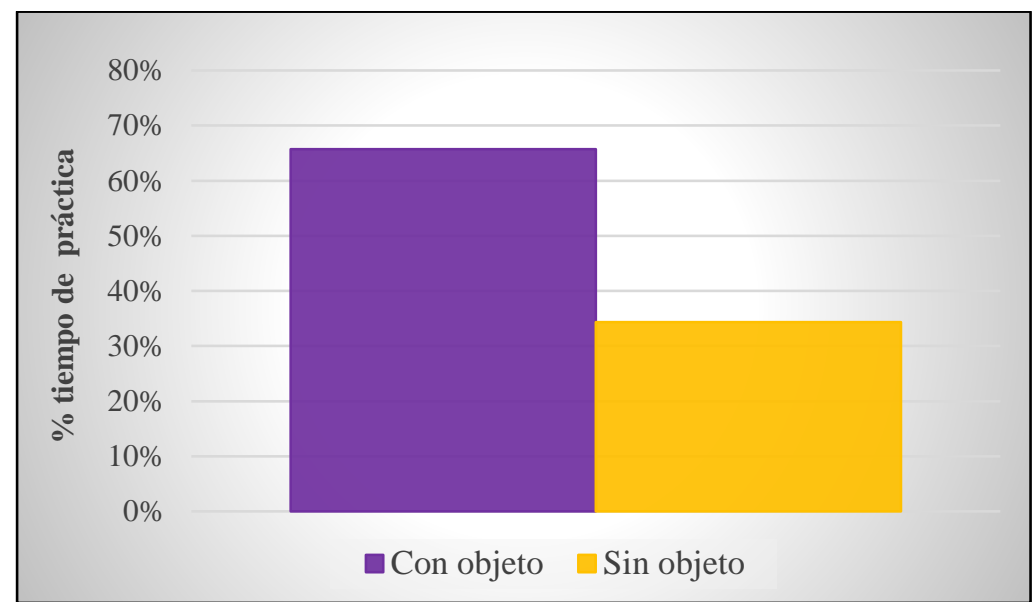

\section{Discusión de los resultados}

Los resultados muestran que en la educación física de Burgos prevalece la práctica motriz con objeto. Así como han constatado recientes estudios realizados en la Comunidad Autónoma Vasca (SANTEODORO, 2015; LÓPEZ DE SOSOAGA, 2017) y en la Valenciana (GIL, 2017), la institución educativa de Castilla y León también prima actividades que requieren material; de tal modo que el currículo oculto de la educación física de primaria parece reproducir la supremacía de prácticas con material en la actualidad (PARLEBAS, 1999). En consecuencia, la escuela burgalesa transmite la cultura material a las jóvenes generaciones, capacitándoles para integrarse en la sociedad y para adaptarse a las demandas de la vida adulta, si bien podría estar inculcándoles la necesidad de poseer objetos para jugar e incitándoles a valorar el Tener, en contraste con el consumo responsable planteado por el Decreto ley.

Los materiales empleados pertenecen a los centros y son de uso exclusivo de la educación física. Así, se trata de un material de propiedad privada pero de uso común. El alumnado de primaria no se responsabiliza de traer de casa ningún objeto para las clases. Los estudiantes no se implican en la recolección de materiales desechados en su hogar ni conocen el precio de los objetos comerciales, de tal manera que no son incitados a tomar conciencia de la sociedad de consumo (JIMÉNEZ, 1993). Así, la institución educativa de primaria proporciona comodidad, si bien no se compromete con el consumo responsable y la preservación del medio ambiente.

Asimismo, las escuelas burgalesas se ciñen al uso de materiales fabricados industrialmente, específicos del área y comprados en tiendas especializadas en material deportivo. No se reutilizan materiales obtenidos en el entorno natural o social: imperan los materiales manufacturados industrialmente 
con el fin de satisfacer una acción concreta. Además de chocar con la promoción del consumo responsable y con la preservación de la naturaleza, esta decisión de los centros impide que el alumnado de primaria interactúe con materiales que demandan creatividad e improvisación (BLÁNDEZ, 1995).

Si bien la cultura material de las escuelas burgalesas de carácter público y concertado se asemeja considerablemente, también se diferencia en relación con la selección de los contenidos y las características de los materiales. En ambos centros prevalecen las situaciones motrices con objeto, pero su presencia es inferior en el colegio franciscano. Así, sus estudiantes aprenden igualmente un repertorio de juegos y actividades que prescinden de material y, en este sentido, son empujados a valorar el Ser sobre el Tener y a asumir una actitud no consumista afín con su ideario sencillo y ecologista. La escuela pública también plantea promover la valoración y el cuidado del entorno en su PEC, aunque no se refiere directamente a la cuestión del consumo.

Los materiales empleados provienen de un proceso de fabricación industrial y son específicos del área. A pesar de ello, el maestro del colegio concertado recurre a unos pocos ejemplares, multiusos, antiguos y poco sofisticados. Esta decisión podría haberse visto influenciada por una supuesta vocación de las instituciones franciscanas hacia la sencillez y la pobreza, al tiempo que remite al consumo responsable y al cuidado y preservación del medio ambiente proyectados por la Ley. Por el contrario, el especialista del centro público se aprovecha de una mayor variedad de objetos, en su mayoría, nuevos, coloridos y atractivos, para las actividades realizadas en clase.

\section{Conclusión}

En conclusión, podemos afirmar que, en lo que concierne la cultura material de la educación física, la función de reproducción social de la escuela está por encima de las diferencias administrativas que pudieran presentarse entre las instituciones públicas y las concertadas. Asimismo, los centros concertados parecen disponer de mecanismos que les permiten distinguirse de la "normalidad" pública mediante un relato educativo construido a partir de su propia cosmovisión. Dicho discurso matiza también en la acción motriz, dándola, en este caso, un componente franciscano.

Finalmente, desentrañar la relación existente entre el proyecto educativo del centro, público y privado concertado, y la educación física, podría ayudar a los docentes a reflexionar sobre su propia labor educativa, mostrando la presencia del currículo oculto. En ocasiones, se tiene la creencia de que todos los contenidos son buenos per se y que la preocupación principal reside en el "cómo" hay que enseñar (progresiones, 
adecuación del material...) en lugar del "porqué" educativo de las situaciones motrices seleccionadas (PARLEBAS, 2001).

\section{Referencias}

BAUDRILLARD, Jean. La société de consommation. París: Gallimard, 1987.

BLÁNDEZ, Julia. La utilización del material y del espacio en educación física. Barcelona: INDE, 1995. COLEGIO DE EDUCACIÓN INFANTIL Y PRIMARIA PADRE MANJÓN [CEIPPM]. Proyecto educativo de centro. Burgos: Autor, 2009.

COLEGIO PRIVADO CONCERTADO NUESTRA SEÑORA DE LOURDES [CPCNSL]. Proyecto educativo de centro. Burgos: Autor, 2004.

CASTILLA Y LEÓN. CONSERJERÍA DE EDUCACIÓN. Decreto 40/2007, de 3 de mayo, por el que se establece el Currículo de la Educación Primaria en la Comunidad de Castilla y León. Boletín Oficial de Castilla y León (B.O.C. y L.), N² 29, de 4 de mayo de 2007.

ESPAÑA. JEFATURA DEL ESTADO. Ley Orgánica 2/2006, de 3 de mayo, de Educación. Boletín Oficial del Estado (B.O.E.), № 106, de 4 de mayo de 2006.

ESPAÑA. MINISTERIO DE EDUCACIÓN Y CIENCIA. Real Decreto 1513/2006, de 7 de diciembre, por el que se establecen las enseñanzas mínimas de la Educación Primaria. Boletín Oficial del Estado (B.O.E.), $\mathrm{N}^{\mathrm{o}} 293$, de 8 de diciembre de 2006.

ETXEBESTE, Joseba. À cloche-pied: les jeux sportifs traditionnels et la socialisation des enfants basques. Saarbrücken: Éditions universitaires européennes, 2012.

ETXEBESTE, Joseba; URDANGARIN, Clara; LAVEGA, Pere; LAGARDERA, Francisco; ALONSO, José Ignacio. El placer de descubrir en praxiología motriz: la etnomotricidad. Acción Motriz, 15, p. 15-24, 2015. Disponible en: http://www.accionmotriz.com/ Acceso en: 15 de enero 2016.

GIL, Javier. La socialización oculta en educación física. Análisis de la enseñanza de las actividades físicas y deportivas en Meliana (València). 2017. 391 pp. Tese (Doctorado en Actividades físicas y deporte). Facultad de Ciencias de la Actividad Física y del Deporte - Universidad del País Vasco (UPV/EHU). Vitoria-Gasteiz, España, 2017.

JIMÉNEZ, María. Consumo y currículum. Cuadernos de Pedagogía, 218, p. 12-13, 1993.

KIRK, David. Physical Education Futures. London: Routledge, 2010.

LÓPEZ DE SOSOAGA, Alfredo. Discurso y praxis de la Educación Física en el País Vasco en el contexto de la Escuela Primaria. 2017. 577 pp. Tese (Doctorado en Actividades físicas y deporte). Facultad de Ciencias de la Actividad Física y del Deporte - Universidad del País Vasco (UPV/EHU). Vitoria-Gasteiz, España, 2017.

LÓPEZ PASTOR, Víctor Manuel; MONJAS, Roberto; PÉREZ BRUNICARDI, Darío. Buscando 
alternativas a la forma de entender y practicar la educación física escolar. Barcelona: INDE, 2004.

MORACE, Francesco. Contratendencias. Una nueva cultura del consumo. Madrid: Celeste, 1993.

PARLEBAS, Pierre. Les tactiques du corps. In: MARIE-PIERRE, Julien; WARNIER, Jean Pierre (Orgs.). Approches de la culture matérielle. Corps à corps avec l'objet. París: L'Harmattan, 1999, p. 29-43.

PARLEBAS, Pierre. Juegos, deporte y sociedad. Léxico de Praxiología Motriz. Barcelona: Paidotribo, 2001.

PARLEBAS, Pierre. Hostilité et solidarité dans les activités motrices. In: DUGAS, Eric; FERRÉOL, Gilles (Orgs.). Oser l'autre : violence et vulnérabilités scolaires en question. Bruselas: EME InterCommunications, 2015, p. 101-117.

RUIZ OLABUÉNAGA, José Ignacio \& ISPIZUA, María Antonia. La descodificación de la vida cotidiana. Métodos de investigación cualitativa. Bilbao: Universidad de Deusto, 1989.

SANTEODORO, Yolanda. Kurutziaga ikastolako gorputz hezkuntzan giza genero eredu motorrak. 2015. 253 pp. Tese (Doctorado en Actividades físicas y deporte). Facultad de Ciencias de la Actividad Física y del Deporte - Universidad del País Vasco (UPV/EHU). Vitoria-Gasteiz, España, 2015.

URDANGARIN, Clara. Bailando jauzi bajo barras y estrellas: una etnografía Zaspiak Bat Group of Dancers de Reno, Nevada. 2009. 417 pp. Tese (Doctorado en Actividades físicas y deporte). Facultad de Ciencias de la Actividad Física y del Deporte - Universidad del País Vasco (UPV/EHU). Vitoria-Gasteiz, España, 2009.

WARNIER, Jean Pierre. Construire la culture matérielle. L'homme qui pensait avec ses doigts. París: PUF, 1999.

WOODS, Peter. La escuela por dentro: la etnografía en la investigación educativa. Barcelona: Paidós, 1987.

\section{Como citar este artigo}

FRANCO, F.C. La cultura material en la educación física escolar: discurso y praxis. Revista Kinesis, Santa Maria, v. 39, p.01-14, 2021.

* Este trabajo de investigación fue financiado por el Programa de Formación y Perfeccionamiento de Personal Investigador del Departamento de Educación, Universidades e Investigación del Gobierno Vasco (España). 